\title{
Healthcare Costs of Acute and Chronic Pain Associated with a Diagnosis of Herpes Zoster
}

\author{
Robert H. Dworkin, PhD, ${ }^{* \dagger}$ Richard White, PhD, ${ }^{\mathcal{S}}$ Alec B. O’Connor, MD, ${ }^{\ddagger}$ Onur Baser, PhD, , and \\ Kevin Hawkins, PhD
}

OBJECTIVES: To determine the healthcare costs of acute and chronic pain associated with herpes zoster.

DESIGN: Retrospective cohort analysis.

SETTING: Inpatient and outpatient care.

PARTICIPANTS: Patients were selected from Medicare, commercial insurance, and Medicaid claims databases if they had a diagnosis of herpes zoster or postherpetic neuralgia (PHN) or were prescribed analgesics after a diagnosis of herpes zoster (possible PHN) and were matched to controls for demographic and clinical factors using propensity scores.

MEASUREMENTS: One-year excess healthcare expenditures attributable to herpes zoster pain or PHN were calculated for inpatient, outpatient, and prescription drug services.

RESULTS: For the Medicare cohort, the average excess cost per patient was $\$ 1,300$ in the year after a diagnosis of herpes zoster with 30 days or fewer of analgesic use and ranged from $\$ 2,200$ to $\$ 2,300$ per patient with $\mathrm{PHN}$ or possible PHN. Patients with possible PHN were 53\% more prevalent than patients with PHN in the Medicare cohort and accounted for half of all excess expenditures. Findings were similar in the younger cohorts with commercial insurance and Medicaid except that costs attributable to PHN and possible PHN were higher, and patients with possible PHN were three to five times as prevalent as patients with PHN.

CONCLUSION: Healthcare costs associated with PHN were substantially greater than those associated with herpes zoster pain that resolved within 30 days. The data suggest that as many as $80 \%$ of patients with PHN may not be diagnosed with PHN and that these patients account for at least half of PHN expenditures. J Am Geriatr Soc 55:11681175, 2007.

From the Departments of *Anesthesiology, ${ }^{\dagger}$ Neurology, ${ }^{\ddagger}$ Medicine, School of Medicine and Dentistry, University of Rochester, Rochester, New York; ${ }^{\S}$ Endo Pharmaceuticals, Chadds Ford, Pennsylvania; "Thomson-Medstat, Ann Arbor, Michigan; and "IMS Health, Ann Arbor, Michigan.

Address correspondence to Dr. Robert H. Dworkin, Department of Anesthesiology, University of Rochester School of Medicine and Dentistry, 601 Elmwood Avenue, Box 604, Rochester, NY 14642.

E-mail: robert_dworkin@urmc.rochester.edu

DOI: $10.1111 / \mathrm{j} .1532-5415.2007 .01231 . x$
Key words: herpes zoster; postherpetic neuralgia; acute pain; chronic pain; healthcare costs

$\mathbf{R}$ eactivation of varicella-zoster virus and its spread from a sensory ganglion to the corresponding dermatome causes herpes zoster (shingles). ${ }^{1,2}$ Herpes zoster occurs annually in approximately one million people in the United States, during the lifetimes of up to $30 \%$ of the population, and in as many as $50 \%$ of those living until 85 years of age. ${ }^{1,3-8}$ The appearance of a characteristic unilateral dermatomal rash with localized pain marks the onset of herpes zoster in nearly all patients. Pain persists after rash healing in a substantial percentage of patients with herpes zoster, although the precise figure depends on age and other risk factors. A recent observational study of patients with herpes zoster (more than half aged $\geq 50$ ) presenting to family physicians in England reported that pain persisted in $38 \%$ at 6 weeks, $27 \%$ at 12 weeks, $16 \%$ at 6 months, and $9 \%$ at 1 year. $^{9}$

The results of recent research provide support for a distinction between three phases of pain associated with herpes zoster - acute pain occurring within 30 days after rash onset; postherpetic neuralgia (PHN), defined as pain that persists 120 days or more after rash onset; and subacute herpetic neuralgia (SHN), pain that persists beyond 30 days but resolves before the diagnosis of PHN is made. ${ }^{10-12} \mathrm{PHN}$ is the most common and most feared complication of herpes zoster in immunocompetent patients, and estimates of its prevalence in the United States range from 500,000 to $1,000,000 . .^{13,14}$ In one large sample, $12.5 \%$ of patients with herpes zoster developed PHN defined as pain 90 days after rash onset of at least 3 on a pain intensity scale of 0 to $10 .{ }^{8}$

It was recently found that the average costs associated with PHN and SHN ranged from $\$ 2,700$ to $\$ 9,300$ in the year after diagnosis, depending on the patient's type of insurance. ${ }^{15}$ The absence of data on the costs of acute pain in patients with herpes zoster (pain that resolved within 30 days after the diagnosis), modest sample sizes in some 
groups, and the need to extrapolate Medicaid costs to allow comparison with other insurance types limited this analysis. The objectives of the present analyses are to evaluate the healthcare costs associated with acute and chronic pain in patients with herpes zoster in an expanded database that addresses these limitations. Accurate determination of the healthcare expenses associated with herpes zoster will increase the validity of cost-effectiveness analyses of treatments for $\mathrm{PHN}^{16}$ and of strategies to prevent herpes zoster and PHN, ${ }^{17}$ for example, zoster vaccination. ${ }^{8}$

\section{METHODS}

This study is a retrospective cohort analysis. Excess healthcare costs in patients with herpes zoster acute pain, PHN, or SHN were calculated by comparing expenditures for cohorts of patients with these painful conditions with expenditures of control groups without these conditions who were propensity score matched on demographic characteristics and comorbidities to the patients with pain to eliminate measurable differences between the groups. Estimates are provided for those with Medicare, commercial insurance, and Medicaid separately.

\section{Study Population}

The data for this study came from Thomson-Medstat's MarketScan suite of databases (www.medstat.com), which was chosen, because it contains individual-level healthcare claims information from Medicare, Medicaid, large employers, and managed care organizations and captures the full continuum of care for nearly 28 million covered lives in all settings, including physician office visits; hospital stays; retail, mail order, and specialty pharmacies; and any insured services managed by specialty vendors. It contains complete information on outpatient prescriptions and high-quality coding and has strong longitudinal tracking at the patient level, with the capability of following more than half of the individuals in MarketScan for 3 years. Three separate databases were used in this study: the Medicare, Commercial Claims and Encounters, and Medicaid databases. The Medicare database contains the healthcare experience of approximately one million individuals with Medicare supplemental insurance paid for by employers. The Medicare-covered portion of payment (represented as Coordination of Benefits Amount) and the employer-paid portion are included in this database. The commercial database contains the healthcare experience of approximately 8 million employees and their dependents (annually) covered under a variety of fee-for-service and capitated health plans, including preferred provider organizations, point of service plans, indemnity plans, and health maintenance organizations. The Medicaid database contains the pooled healthcare experience of approximately 10 million Medicaid enrollees from several geographically dispersed states. All these databases link enrollment and medical claims for inpatient, outpatient, and outpatient prescription drug services for each patient.

Patients were considered to have herpes zoster acute pain if they had a healthcare claim with primary diagnosis of herpes zoster (International Classification of Diseases, 10th Revision (ICD-10) 053.9x, 053.0x, 053.10, 053.11, $053.2 \mathrm{x}, 053.7 \mathrm{x}, 053.8 \mathrm{x})$ and had received $<31$ days of analgesic medication starting within 30 days after the herpes zoster diagnosis. Medications considered analgesics were those commonly used during the study period, whether appropriately or not, in the treatment of PHN and other neuropathic pain conditions, including gabapentin, lidocaine patch $5 \%$, opioid analgesics (e.g., buprenorphine, butorphanol, codeine, dezocine, dihydrocodeine, fentanyl, hydrocodone, hydromorphone, levorphanol, meperidine, methadone, morphine sulfate, nalbuphine, oxycodone, oxymorphone, pentazocine, propoxyphene) alone and in combination with other medications, tramadol, tricyclic antidepressants (TCAs; amitriptyline, amoxapine, clomipramine, desipramine, doxepin, imipramine, nortriptyline, protriptyline, trimipramine), other anticonvulsants (lamotrigine, oxcarbazepine), other antidepressants (bupropion, citalopram, escitalopram, fluoxetine, fluvoxamine, isocarboxazid, maprotiline, mirtazepine, nefazodone, paroxetine, phenelzine, sertraline, tranylcypromine, trazodone, venlafaxine), and benzodiazepines (alprazolam, chlordiazepoxide, clorazepate, diazepam, estazolam, flurazepam, halazepam, lorazepam, midazolam, oxazepam, prazepam, quazepam, temazepam, triazolam). ${ }^{18,19}$ Because antidepressants other than TCAs and benzodiazepines may not have been used for pain associated with herpes zoster, considering these medications to be analgesics may have introduced a misclassification bias; for this reason, we performed sensitivity analyses that excluded these medications.

Patients were considered to have PHN if they had a healthcare claim with a primary diagnosis of PHN (ICD-10 $053.12,053.13,053.19)$. Because the concept of subacute herpetic neuralgia has only been validated in the past several years, ${ }^{10-12}$ it is likely that this group includes patients with SHN who were diagnosed with PHN.

Patients were considered to have possible PHN if they had a healthcare claim with a primary diagnosis of herpes zoster (ICD-10 053.9x, 053.0x, 053.10, 053.11, 053.2x, $053.7 \mathrm{x}, 053.8 \mathrm{x})$ and had received more than 30 days of analgesic medication starting within 30 days after the herpes zoster diagnosis. As above, medications considered to be analgesics in the primary analysis were those commonly used during the study period, whether appropriately or not, in the treatment of PHN and other neuropathic pain conditions. ${ }^{18,19}$ Because of their persisting use of analgesic medications after their diagnosis of herpes zoster, it is likely that the possible PHN group consists of a mixture of patients with PHN and SHN whose herpes zoster diagnoses were not revised to reflect their persistent pain.

The date of the herpes zoster or PHN diagnosis that met the above criteria was considered the index event. The 6 months preceding the index date was the pre-period, which was used for propensity matching, and the 12 months after the index date was the post-period, from which expenditure data were derived. The index date was between July 1, 2001, and December 31, 2003.

Patients were only included in the study once. If a patient was eligible to be in more than one of the pain cohorts, they were assigned based on the following hierarchy: PHN, possible PHN, herpes zoster (i.e., if a patient with herpes zoster later developed PHN, they were included in the PHN cohort). It is important to emphasize that all prescription medication claims are included in the Medicare, commer- 
cial insurance, and Medicaid databases and that the only way that patients could have obtained prescription analgesics without being identified would have been if they did not use their insurance and paid cash for the prescription.

Patients were excluded from the study if they were not continuously enrolled from the pre-period through the postperiod or if at any time during the study they were pregnant or insured under a capitated insurance plan. Pregnant patients were excluded, because certain pain medications may be contraindicated during pregnancy. Healthcare payment information on capitated insurance claims is often missing or not representative of the services provided; thus patients with capitated coverage were excluded.

A random sample of patients without diagnoses of herpes zoster and PHN were the control cohorts. Separate control groups were selected for each pain cohort (herpes zoster, possible PHN, and PHN) and insured group (Medicare, commercial, and Medicaid). The control groups were nine times as large as the corresponding pain cohorts and met the same exclusion criteria. The index date for the controls was randomly assigned from the appropriate pain cohort.

\section{Measurements}

Patient demographic characteristics included age at index diagnosis and sex. A residence variable (urban vs rural) was also included and defined based on residence within a metropolitan statistical area. The region of the country where the patient resided was also tabulated for the Medicare and commercial patients (this information was not available for Medicaid patients). The regions included the northeast, north central, south, and west. Because patients' index dates could vary from 2001 to 2003, this was controlled for by including binary indicators for each year. For the Medicaid patients, race/ethnicity was also available and consisted of African American, American Indian or Alaskan native, Asian or Pacific Islander, Caucasian, Hispanic, and other/missing.

To control for overall medical comorbidity, the Charlson Comorbidity Index (CCI) score in the pre-period was used. The CCI score is a measure of the burden of comorbid illness that can be calculated easily with claims data. Higher $\mathrm{CCI}$ values indicate a higher probability of death or major disability due to comorbid illness. ${ }^{20,21}$

To control for concomitant psychiatric conditions, a psychiatric diagnostic group (PDG) variable was used that measures the number of major psychiatric conditions for which a patient received treatment in the pre-period. ${ }^{22}$ This is based on ICD-9 diagnosis codes present on claims, which are analogous to the major diagnostic categories used in the diagnosis-related groups classification system.

Medicare and commercial patients were divided into those covered by traditional indemnity plans and those covered by managed care plans. Managed care plans included point of service and preferred provider service plans.

The outcome variable of interest in this study was annual healthcare expenditures, which were measured for each patient in the post-period. Excess healthcare costs were estimated as the difference in average annual healthcare expenditures between the pain cohorts and the matched control groups. Expenditures are reported in 2004 dollars.

\section{Data Analyses}

Descriptive analyses were used to compare the pain cohorts with their respective control groups before and after matching. Chi-square tests were used to compare binary indicators across the cohorts, and Student $t$ tests were used for the continuous variables.

A key problem with observational studies is the lack of randomization in assigning individuals to groups. Because of this, the existence of confounding factors may bias results from descriptive analyses. Propensity score matching was developed for adjusting such confounding factors. The main purpose of matching is to reduce selection bias by improving the balance between groups. Propensity score matching employs the predicted probability of group membership based on observed predictors. For example, given two patients, one in a pain cohort and one in its control group with the same (or a similar) propensity score, these patients can be considered to be randomly assigned to each group. $^{23}$

The control patients were matched to pain cohorts according to the predicted probability of being in that pain cohort. This probability was estimated for each patient based on a logistic regression analysis that controlled for patient demographic characteristics (age, sex, insurance type, region, urban residency, and index year, and in addition, race for the Medicaid patients) and overall comorbidities (CCI and PDG) in the pre-period. This was done separately for each pain cohort and each insured group (Medicare, commercial, Medicaid), creating a total of nine pain cohort and control group pairs. Patients were matched using a nearest-neighbor, within-caliber, one-to-one approach. ${ }^{24}$ Patients who were not matched were eliminated from the sample.

Consistent with previous studies, ${ }^{25}$ a second-stage regression was estimated to control for remaining significant differences between the cohorts on the matching variables. Specifically, the second-stage regression used total healthcare expenditures in the post-period as the dependent variable and the same independent variables used in the matching. Because healthcare expenditures are often skewed, a generalized linear model was used. When normality does not hold, generalized linear models use quasimaximum likelihood estimation rather than maximum likelihood estimation. These estimators are robust to distributional misspecification, that is, if the conditional mean is correctly specified, quasi-maximum likelihood consistently estimates the parameters regardless of distribution.

Although benzodiazepines and antidepressants other than TCAs are used to treat patients with PHN and other neuropathic pain conditions, ${ }^{19}$ their use in neuropathic pain is not evidence based and may not be as widespread as treatments for which there is evidence of efficacy. ${ }^{18}$ For this reason, sensitivity analyses were conducted in which benzodiazepines and "other antidepressants" were deleted from the list of analgesics used for determining which patients with herpes zoster pain to include in the study. All of the models were rerun, and the results of these sensitivity 
analyses were compared with the primary analyses that included benzodiazepines and "other antidepressants."

\section{RESULTS}

Post-matching demographic and clinical characteristics of patients and controls are shown in Table 1. (Additional preand post-matching characteristics for each of the pain and control cohorts are available from the first author.) After matching, patients in the Medicare group were approximately 25 years older than patients in the commercial and Medicaid groups. Medicare patients also had higher CCI scores than those with commercial insurance and lower mean numbers of PDGs than those with Medicaid.

For all three types of insurance, patients with herpes zoster acute pain were well matched with their control groups except that CCI scores were higher in the pain cohorts than in their respective control groups (Table 1). Patients with PHN and possible PHN were generally well matched with their control groups for all insurance types except that CCI scores and number of PDGs were higher in the pain cohorts than in their respective control groups (Table 1).

\section{Multivariate Results}

The expenditure data for each type of pain for the three insurance types are presented in Table 2. Exact coefficients and marginal effects for the generalized linear models are available from the first author.

\section{Herpes Zoster Acute Pain}

The annual excess healthcare costs per patient associated with herpes zoster acute pain were $\$ 1,298$ in the Medicare sample $(P<.001)$. Excess expenditure estimates were similar in the commercial sample but were $42 \%$ lower in the Medicaid sample (Table 2). For each insurance type, greater comorbidity, as assessed according to the CCI and PDG scores, and older age were associated with greater expen-

\begin{tabular}{|c|c|c|c|c|c|c|c|c|c|}
\hline Characteristic & $\begin{array}{l}\text { Herpes } \\
\text { Zoster }\end{array}$ & $\begin{array}{l}\text { Herpes Zoster } \\
\text { Controls }\end{array}$ & $\begin{array}{c}P- \\
\text { Value }\end{array}$ & PHN & $\begin{array}{c}\text { PHN } \\
\text { Controls }\end{array}$ & $\begin{array}{c}P- \\
\text { Value }\end{array}$ & $\begin{array}{l}\text { Possible } \\
\text { PHN }\end{array}$ & $\begin{array}{l}\text { Possible PHN } \\
\text { Controls }\end{array}$ & $\begin{array}{c}P \text { - } \\
\text { Value }\end{array}$ \\
\hline \multicolumn{10}{|l|}{ Medicare } \\
\hline \multicolumn{10}{|l|}{ Demographics } \\
\hline $\mathrm{n}$ & 2,554 & 2,554 & & 1,722 & 1,722 & & 2,634 & 2,634 & \\
\hline Age & 75.2 & 75.2 & .80 & 77.1 & 77.2 & .58 & 75.9 & 76.1 & .43 \\
\hline Female, \% & 61.1 & 62.2 & .42 & 66.0 & 66.4 & .80 & 70.3 & 72.0 & .18 \\
\hline \multicolumn{10}{|l|}{ Insurance type, \% } \\
\hline Missing/unknown & 0.08 & 0.00 & .16 & 0.00 & 0.12 & .16 & 0.11 & 0.00 & .08 \\
\hline Indemnity & 57.7 & 62.3 & .001 & 58.2 & 59.7 & .37 & 59.1 & 61.6 & .06 \\
\hline POS or PPO & 42.2 & 37.7 & .001 & 41.8 & 40.2 & .33 & 40.8 & 38.4 & .08 \\
\hline \multicolumn{10}{|l|}{ Health status } \\
\hline $\mathrm{CCl}$ & 1.41 & 1.27 & .009 & 1.70 & 1.54 & .01 & 1.85 & 1.57 & $<.001$ \\
\hline PDGs & 0.07 & 0.08 & .36 & 0.17 & 0.11 & .001 & 0.22 & 0.12 & $<.001$ \\
\hline \multicolumn{10}{|l|}{ Commercial insurance } \\
\hline \multicolumn{10}{|l|}{ Demographics } \\
\hline $\mathrm{n}$ & 4,696 & 4,696 & & 1,037 & 1,037 & & 3,285 & 3,285 & \\
\hline Age & 48.8 & 48.8 & .94 & 52.8 & 53.0 & .75 & 51.7 & 52.2 & .04 \\
\hline Female, \% & 60.3 & 60.1 & .88 & 66.3 & 66.9 & .78 & 73.2 & 72.8 & .68 \\
\hline \multicolumn{10}{|l|}{ Insurance type } \\
\hline Missing/unknown & 0.06 & 0.13 & .32 & 0.39 & 0.19 & .41 & 0.09 & 0.06 & .66 \\
\hline Indemnity & 19.5 & 19.8 & .74 & 22.5 & 21.6 & .63 & 24.0 & 23.5 & .60 \\
\hline POS or PPO & 80.5 & 80.1 & .68 & 77.1 & 78.2 & .56 & 75.9 & 76.5 & .58 \\
\hline \multicolumn{10}{|l|}{ Health status } \\
\hline $\mathrm{CCl}$ & 0.57 & 0.47 & $<.001$ & 0.87 & 0.70 & .003 & 0.93 & 0.69 & $<.001$ \\
\hline PDGs & 0.12 & 0.09 & .003 & 0.24 & 0.14 & $<.001$ & 0.40 & 0.25 & $<.001$ \\
\hline \multicolumn{10}{|l|}{ Medicaid } \\
\hline \multicolumn{10}{|l|}{ Demographics } \\
\hline $\mathrm{n}$ & 1,407 & 1,407 & & 373 & 373 & & 1,872 & 1,872 & \\
\hline Age & 45.1 & 45.8 & .33 & 50.3 & 53.9 & .003 & 49.4 & 51.5 & $<.001$ \\
\hline Female, \% & 62.0 & 62.5 & .76 & 65.4 & 63.5 & .59 & 71.4 & 67.5 & .01 \\
\hline \multicolumn{10}{|l|}{ Health status } \\
\hline $\mathrm{CCl}$ & 1.28 & 1.00 & $<.001$ & 1.53 & 1.06 & $<.001$ & 1.92 & 1.48 & $<.001$ \\
\hline PDGs & 0.40 & 0.44 & .15 & 0.74 & 0.57 & .020 & 1.18 & 0.91 & $<.001$ \\
\hline
\end{tabular}

$\mathrm{PHN}=$ postherpetic neuralgia; $\mathrm{POS}=$ point of service plan; $\mathrm{PPO}=$ preferred provider organization plan; CCI = Charlson Comorbidity Index; PDGs = psychiatric diagnosis group diagnoses. 
Table 2. Total Excess Expenditures Derived from the Generalized Linear Models

\begin{tabular}{|c|c|c|c|c|c|c|}
\hline \multirow[b]{2}{*}{ Pain Type } & \multicolumn{2}{|c|}{ Medicare } & \multicolumn{2}{|c|}{ Commercial } & \multicolumn{2}{|c|}{ Medicaid } \\
\hline & Expenditures & $P$-Value & Expenditures & $P$-Value & Expenditures & $P$-Value \\
\hline PHN & $\$ 2,159$ & $<.001$ & $\$ 5,387$ & $<.001$ & $\$ 5,112$ & $<.001$ \\
\hline Possible PHN & $\$ 2,292$ & $<.001$ & $\$ 5,742$ & $<.001$ & $\$ 5,178$ & $<.001$ \\
\hline
\end{tabular}

$\mathrm{PHN}=$ postherpetic neuralgia.

ditures $(P>.001)$, although the magnitude of the effect for age was modest.

\section{PHN and Possible PHN}

For Medicare patients, the annual excess healthcare costs associated with PHN and possible PHN were \$2,159 and $\$ 2,292$, respectively $(P<.001)$. Based on these estimates, excess expenditures for patients with $\mathrm{PHN}$ and possible PHN were more than twice as high in patients with commercial insurance and Medicaid as in Medicare patients (Table 2).

For each insurance type, higher CCI scores and greater number of PDGs were associated with greater expenditures in patients with PHN and possible PHN $(P<.001)$. The majority of patients with possible PHN were taking analgesics between 4 and 6 months after the diagnosis of herpes zoster: $79.2 \%$ in the Medicare sample (vs $32.6 \%$ of controls), $82.9 \%$ in the commercial sample (vs $30.6 \%$ of controls), and $88.5 \%$ in the Medicaid sample (vs $49.1 \%$ of controls).

In the Medicare sample, 53\% more patients had possible PHN than had PHN (Table 1). Patients with possible PHN were also more prevalent in the commercial and Medicaid samples. Because it was prevalent and expensive to treat, possible PHN accounted for the majority of total expenditures (Figure 1).

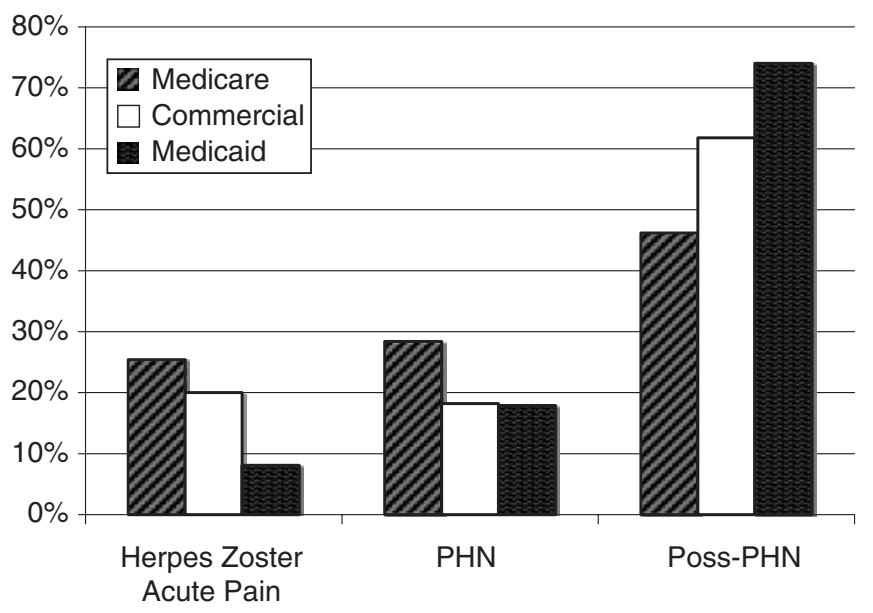

$\mathrm{PHN}=$ postherpetic neuralgia;

Poss-PHN = possible postherpetic neuralgia.

Figure 1. Percentages of all expenditures accounted for by different pain conditions (reflects costs per patient and number of patients in each category).

\section{Expenditure Components}

Table 3 displays the percentages of the total expenditures attributable to different types of care for each pain category and insurance. In all of the cohorts, the majority of expenses are not directly attributable to herpes zoster or mental health (but occur in the "other" category). For each insurance type, expenditures for prescriptions are substantially greater for patients with PHN and possible PHN than for patients with herpes zoster with acute pain. For patients with Medicare and commercial insurance, approximately half of all healthcare payments represent outpatient nonemergency department claims.

\section{Sensitivity Analyses}

When benzodiazepines and "other antidepressants" were deleted from the list of analgesics used for determining which patients with herpes zoster pain to include in the study, the numbers of Medicare patients in the herpes zoster acute pain and possible PHN cohorts decreased $12 \%$ and $10 \%$, respectively, and the respective expenditure estimates decreased $32 \%$ and $20 \%$. The numbers of patients in the different commercial insurance and Medicaid cohorts decreased $12 \%$ to $16 \%$, with the expenditure estimates falling $40 \%$ to $47 \%$ for commercial patients and $69 \%$ to $87 \%$ for Medicaid patients (specific results for each of the pain and control cohorts are available from the first author). The results for the PHN models did not change, because medication use was not a criterion for being included in this cohort.

\section{DISCUSSION}

The results indicate that annual healthcare costs associated with PHN range from $\$ 2,159$ to $\$ 5,387$ and are much greater than the healthcare costs associated with acute pain in patients with herpes zoster, which range from $\$ 757$ to $\$ 1,313$. Patients who were not diagnosed with PHN but who were classified as having possible PHN generated most of the excess expenditures. These patients had diagnoses of herpes zoster but had received more than 30 days of analgesic medication starting within 30 days after their diagnosis with herpes zoster. Their annual healthcare costs ranged from $\$ 2,292$ to $\$ 5,742$ and were similar to those of the patients who were diagnosed with PHN.

The total costs of care and the components of the costs of care (Table 3) associated with PHN and possible PHN were remarkably similar. Furthermore, $79.2 \%$ to $88.5 \%$ of the patients with possible PHN were taking analgesics more than 3 months after their diagnosis with herpes zoster. Considered together, these data suggest that a large 
Table 3. Excess Expenditures Attributable to Different Types of Care in the Post-Matching Cohorts*

\begin{tabular}{lrcr}
\hline & Medicare & Commercial & Medicaid \\
\cline { 2 - 4 } \multicolumn{1}{c}{ Type of Care } & \multicolumn{3}{c}{$\%$} \\
\hline Herpes zoster acute pain & & & \\
Outpatient ED claims & 3 & 54 & 11 \\
Outpatient non-ED claims & 58 & 17 & -16 \\
Inpatient & 31 & 23 & 76 \\
Prescriptions & 8 & & 28 \\
PHN & & 2 & \\
Outpatient ED claims & 2 & 44 & -21 \\
\hline Outpatient non-ED claims & 48 & 26 & 35 \\
Inpatient & 22 & 28 & 83 \\
\hline Prescriptions & 28 & & \\
Possible PHN & & 3 & 3 \\
Outpatient ED claims & 3 & 41 & 16 \\
Outpatient non-ED claims & 39 & 18 & 15 \\
\hline Inpatient & 20 & 39 & 65 \\
\hline Prescriptions & 38 & & \\
\hline
\end{tabular}

* Negative percentages occur when total expenditures in the control cohort exceed those of pain patients for a particular component of care; total percentages can differ from $100 \%$ because of rounding.

$\mathrm{PHN}=$ postherpetic neuralgia; $\mathrm{ED}=$ emergency department.

percentage of the patients who were classified with possible PHN probably had undiagnosed $\mathrm{PHN}$, that is, these patients had developed PHN but had not had their diagnoses changed from herpes zoster to reflect the persistence of their pain. It is important to recognize that the group of patients with possible PHN was much larger than the group with PHN and that much of the total healthcare expenditure burden associated with persisting pain in patients with herpes zoster would be missed if patients without a diagnosis of PHN but with a diagnosis of herpes zoster were excluded from consideration. The proportion of patients with possible PHN was somewhat lower in the Medicare group, which suggests that Medicare patients with persisting pain are more likely to be diagnosed with PHN than patients with commercial insurance or Medicaid (Table 1). This could reflect greater familiarity with PHN among providers of Medicare patients or greater expectation of PHN in older patients. Future research must consider the effect of underdiagnosis of PHN not only on estimates of healthcare costs, but also on the adequacy of clinical care in the community.

In these data, older age was associated with higher expenditures for many of the pain cohorts, although the magnitude of this effect was modest. However, even though the average Medicare patient was considerably older than the average patient with commercial insurance or Medicaid, Medicare expenditures were generally lower than commercial insurance or Medicaid expenditures for the cohorts with PHN and possible PHN. The explanation for this is unclear but could involve higher background healthcare expenditures in the Medicare control groups and possibly greater out-of-pocket expenses for Medicare patients.
Patients with PHN suffer from physical disability and emotional distress and have greatly increased healthcare utilization as a result of their chronic pain. ${ }^{26-28}$ In addition, acute pain in herpes zoster has been shown to have adverse effects on physical and emotional functioning. ${ }^{29,30}$ On the basis of a cost-utility analysis conducted for England and Wales, ${ }^{31}$ the total societal costs of herpes zoster and PHN in the United States could be estimated as approximately $\$ 1.7$ billion per year, which would include physician visits, hospitalizations, work loss, and household expenditures. The percentages of these costs that are attributable separately to herpes zoster and PHN cannot be estimated from this study. In studies conducted in the 1990s, the healthcare costs of PHN were estimated to be as high as $\$ 3,250$ per year (converted to 2004 dollars). ${ }^{26,32-35}$ A recent study of the economic costs of neuropathic pain, including PHN, reported that calendar year 2000 healthcare charges were higher in patients with neuropathic pain $(\$ 17,355)$ than in matched controls $(\$ 5,715)$ in a sample of patients drawn from private healthcare plans in the United States. ${ }^{36}$ Most recently, using a smaller set of data with no information on acute pain during herpes zoster, markedly variable estimates of excess healthcare costs associated with PHN (including SHN) were found that ranged from $\$ 2,696$ to $\$ 9,310$ during the first year, depending on insurance type. ${ }^{15}$

The present study is the first assessment of healthcare costs associated with the acute pain of herpes zoster and the chronic pain of PHN in the United States. The present analysis extends earlier findings by providing more-precise estimates of expenditures for each of three insurance types; separate estimates for patients with herpes zoster acute pain, patients diagnosed with $\mathrm{PHN}$, and patients who received more than 30 days of analgesics after a diagnosis of herpes zoster, who appear to be undiagnosed patients with PHN; and quantification of the relative frequency of these different types of pain after a diagnosis of herpes zoster and their relative contributions to the overall cost burden of pain associated with herpes zoster.

The incidence of herpes zoster seems to have increased in the past several decades, ${ }^{37}$ and several factors suggest that the number of patients suffering from herpes zoster and PHN will continue to increase. The number of individuals at the greatest risk for herpes zoster-because they are older or immunocompromised - is expected to rise, and older age also increases the risk of PHN. ${ }^{27}$ In addition, recent epidemiological data ${ }^{38,39}$ are consistent with the prediction that the incidence of herpes zoster will increase because childhood varicella vaccination has reduced the opportunities for subclinical immune boosting associated with exposure to varicella-zoster virus. ${ }^{40-42}$

These analyses in the present study have several limitations. Accurate categorization of insurance information depends on correct ICD-10 coding, and it was not possible to evaluate the accuracy of the diagnoses used. It is also possible that cases of herpes zoster acute pain and possible PHN could have been missed and that the costs of these conditions underestimated if analgesics were paid for using other means than insurance, either because of inadequate coverage or use of over-the-counter medications. It is likely that including patients using only over-the-counter medications would increase the number of patients classified as having pain, but if such patients are prevalent, the resulting 
estimates of per patient excess expenditures would be decreased; furthermore, use of milder analgesics to identify patients with herpes zoster pain and PHN could cause misclassification of other types of pain, such as from headache or arthritis. Although the results of the sensitivity analyses suggest that the expenditure estimates for herpes zoster acute pain and possible PHN differ depending on the medications considered to be analgesics, the Medicare results were the least affected. Furthermore, the identification of PHN cases was based solely on ICD-10 codes, so the estimates of PHN-related expenditures are not dependent on which medications were considered to be analgesics.

Propensity score matching, although powerful, cannot account for all possible differences that might affect healthcare expenditures. For example, it is possible that the outcomes of patients with the same observable characteristics could vary widely because of some unobservable factors, such as physician or practice prescribing patterns. Moreover, only direct insurance expenditures were measured, which underestimates the total cost to society of herpes zoster pain. It is likely that acute and chronic pain in patients with herpes zoster are associated with significant out-of-pocket expenses and additional societal costs, such as lost work time, as well as substantial impairment in quality of life, none of which are included in the present estimates and all of which would increase the overall costs of herpes zoster. ${ }^{43}$ Finally, the estimates of healthcare expenditures capture excess expenses only during the first year after a diagnosis of herpes zoster; the total cost of care delivered in subsequent years therefore remains unknown.

Our results can provide a basis for cost-effectiveness analyses ${ }^{16}$ of existing treatments for PHN. ${ }^{44}$ Because many patients with PHN are completely or partially refractory to these treatments ${ }^{45}$ the development of interventions to prevent herpes zoster and PHN has become a research priority. The data from the current study can also be used in cost-effectiveness analyses of such preventive interventions, ${ }^{17,46}$ which include vaccination of older individuals ${ }^{8}$ and antiviral therapy and other treatments designed to reduce the risk of PHN in patients with herpes zoster. ${ }^{47}$

\section{ACKNOWLEDGMENTS}

Financial Disclosure: Funded by grants from Endo Pharmaceuticals to the University of Rochester Office of Professional Education and to Thomson-Medstat. Robert H. Dworkin has received research support, consulting fees, or honoraria in the past year from Allergan, Cephalon, CombinatoRx, Dara, Dov, Eli Lilly, Endo, EpiCept, Fralex, GW Pharmaceuticals, Johnson \& Johnson, Merck, NeurogesX (also stock options), Novartis, Pfizer, Schwarz Pharma, Supernus, the U.S. Food and Drug Administration, the National Institutes of Health, the U.S. Department of Veterans Affairs, Wyeth, and XTL Biopharmaceuticals. Richard White is employed by Endo Pharmaceuticals. Alec B. O'Connor is supported in part by a grant from the National Institute on Aging (T32-AG020493-02). Onur Baser is employed by Thomson-Medstat. Kevin Hawkins was employed by Thomson-Medstat and is currently employed by IMS Health.
Author Contributions: All authors participated in study concept and design, data acquisition, analysis and interpretation of data, and preparation of the manuscript. Drs. Baser and Hawkins had primary responsibility for performing the statistical analyses.

Sponsor's Role: Richard White, an employee of the sponsor (Endo Pharmaceuticals), participated in study design, interpretation of results, and manuscript preparation. The sponsor manufactures lidocaine patch $5 \%$, which is approved by the U.S. Food and Drug Administration for the treatment of postherpetic neuralgia, but this article involves healthcare costs of postherpetic neuralgia and not specific treatments for this condition.

\section{REFERENCES}

1. Hope-Simpson RE. The nature of herpes zoster: A long-term study and a new hypothesis. Proc R Soc Med 1965;58:9-20.

2. Gnann JW Jr, Whitley RJ. Herpes zoster. N Engl J Med 2002;347:340-346.

3. Hope-Simpson RE. Postherpetic neuralgia. J R Coll Gen Pract 1975;25: $571-575$.

4. Donohue JG, Choo PW, Manson JE et al. The incidence of herpes zoster. Arch Intern Med 1995;155:1605-1609.

5. Brisson M, Edmunds WJ, Law B et al. Epidemiology of varicella zoster virus infection in Canada and the United Kingdom. Epidemiol Infect 2001;127: 305-314.

6. Brisson M, Edmunds WJ. Epidemiology of varicella-zoster virus in England and Wales. J Med Virol 2003;70:S9-S14.

7. Chapman RS, Cross KW, Fleming DM. The incidence of shingles and its implications for vaccination policy. Vaccine 2003;21:2541-2547.

8. Oxman MN, Levin MJ, Johnson GR et al. A vaccine to prevent herpes zoster and postherpetic neuralgia in older adults. N Engl J Med 2005;352: 2271-2284.

9. Scott FT, Leedham-Green ME, Barret-Muir WY et al. A study of shingles and the development of postherpetic neuralgia in east London. J Med Virol 2003;70(Suppl 1):S24-S30.

10. Arani RB, Soong SJ, Weiss HL et al. Phase specific analysis of herpes zoster associated pain data: A new statistical approach. Stat Med 2001;20: 2429-2439.

11. Desmond RA, Weiss HL, Arani RB et al. Clinical application for change-point analysis of herpes zoster pain. J Pain Symptom Manage 2002;23:510-516.

12. Jung BF, Johnson RW, Griffin DRJ et al. Risk factors for postherpetic neuralgia in patients with herpes zoster. Neurology 2004;62:1545-1551.

13. Bennett GJ. Neuropathic pain: An overview. In: Borsook D, ed. Molecular Neurobiology of Pain. Seattle: IASP Press, 1997, pp 109-113.

14. Bowsher D. The lifetime occurrence of herpes zoster and prevalence of postherpetic neuralgia: A retrospective survey in an elderly population. Eur J Pain 1999;3:335-342.

15. Dworkin RH, White R, O'Connor AB et al. Health care expenditure burden of persisting herpes zoster pain. Pain Med; in press.

16. Cepeda MS, Farrar JT. Economic evaluation of oral treatments for neuropathic pain. J Pain 2006;7:119-128.

17. Hornburger J, Robertus K. Cost-effectiveness of a vaccine to prevent herpes zoster and postherpetic neuralgia in older adults. Ann Intern Med 2006;145:317-325.

18. Dworkin RH, Backonja M, Rowbotham MC et al. Advances in neuropathic pain: Diagnosis, mechanisms, and treatment recommendations. Arch Neurol 2003;60:1524-1534.

19. Oster G, Berger A, Dukes E et al. Use of potentially inappropriate pain-related medications in older adults with painful neuropathic disorders. Am J Geriatr Pharmacother 2004;2:163-170.

20. Charlson ME, Pompei P, Ales KL et al. A new method of classifying prognostic comorbidity in longitudinal studies: Development and validation. J Chronic Dis 1987;40:373-383.

21. D'Hoore W, Bouckaert A, Tilquin C. Practical considerations on the use of the Charlson comorbidity index with administrative data bases. J Clin Epidemiol 1996;49:1429-1433.

22. Ashcraft ML, Fries BE, Nerenz DR et al. A psychiatric patient classification system: An alternative to diagnosis-related groups. Med Care 1989;27: 543-557.

23. Basur O. Too much ado about propensity score models? Comparing methods of propensity score matching. Value Health 2006;9:377-385.

24. Ganguly R, Martin B, Dorfman J et al. In search of an unbiased estimate of treatment effect using observational data: A comparison of propensity scoring 
and Heckman two stage sample selection models. ISPOR Connections 2004;10:2-5.

25. Ozminkowski RJ, Burton W, Goetzel R et al. The impact of rheumatoid arthritis on medical expenditures, absenteeism, and short-term disability benefits. J Occup Environ Med 2006;48:135-148.

26. Davies L, Cossins L, Bowsher D et al. The cost of treatment for post-herpetic neuralgia in the UK. Pharmacoeconomics 1994;6:142-148.

27. Dworkin RH, Schmader KE. Epidemiology and natural history of herpes zoster and postherpetic neuralgia. In: Watson CPN, Gershon AA, eds. Herpes Zoster and Postherpetic Neuralgia, 2nd Ed. New York: Elsevier Press, 2001, pp 39-64.

28. Coplan PM, Schmader K, Nikas A et al. Development of a measure of the burden of pain due to herpes zoster and postherpetic neuralgia for prevention trials: Adaptation of the Brief Pain Inventory. J Pain 2004;5:344-356.

29. Lydick E, Epstein R, Himmelberger D et al. Herpes zoster and quality of life: A self-limited disease with severe impact. Neurology 1995;45(Suppl 8):S52-S53.

30. Katz J, Cooper EM, Walther RR et al. Acute pain in herpes zoster and its impact on health-related quality of life. Clin Infect Dis 2004;39:342-348.

31. Brisson M, Edmunds WJ. Varicella vaccination in England and Wales: Costutility analysis. Arch Dis Child 2003;88:862-869.

32. Paul JE, Mauskopf JA, Bell L. Cost-consequence models for varicella-zoster virus infections. Pharmacotherapy 1995;15:49S-58S.

33. Grüger J, Backhouse ME. Economic evaluation of antiviral therapy for the treatment of herpes zoster in immunocompetent adults. Pharmacoeconomics 1997;11:262-273.

34. Huse DM, Schainbaum S, Kirsch AJ et al. Economic evaluation of famciclovir in reducing the duration of postherpetic neuralgia. Am J Health Syst Pharmacol 1997;54:1180-1184.

35. Smith KJ, Roberts MS. Cost effectiveness of newer antiviral agents for herpes zoster: Is the evidence spotty? J Infect Dis 1998;178(Suppl 1):S85-S90.
36. Berger A, Dukes EM, Oster G. Clinical characteristics and economic costs of patients with painful neuropathic disorders. J Pain 2004;5:143-149.

37. Thomas SL, Hall AJ. What does epidemiology tell us about risk factors for herpes zoster? Lancet Infect Dis 2004;4:26-33.

38. Mullooly JP, Riedlinger K, Chun C et al. Incidence of herpes zoster, 19972002. Epidemiol Infect 2005;133:245-253.

39. Yih WK, Brooks DR, Lett SM et al. The incidence of varicella and herpes zoster in Massachusetts as measured by the Behavioral Risk Factor Surveillance System (BRFSS) during a period of increasing varicella vaccine coverage, 1998-2003. BMC Public Health 2005;5.

40. Schuette MC, Hethcote HW. Modeling the effects of varicella vaccination programs on the incidence of chickenpox and shingles. Bull Math Biol 1999;61:1031-1064.

41. Edmunds WJ, Brisson M. The effect of vaccination on the epidemiology of varicella zoster virus. J Infect 2002;44:211-219.

42. Brisson M, Edmunds WJ, Gay NJ. Varicella vaccination: Impact of vaccine efficacy on the epidemiology of VZV. J Med Virol 2003;70:S31-S37.

43. Grant DM, Mauskopf JA, Bell L et al. Comparison of valaciclovir and acyclovir for the treatment of herpes zoster in immunocompetent patients over 50 years of age: A cost-consequence model. Pharmacotherapy 1997;17: 333-341.

44. Hempenstall K, Nurmikko TJ, Johnson RW et al. Analgesic therapy in postherpetic neuralgia: A quantitative systematic review. PLoS Med 2005; 2:0628-0644.

45. Watson CPN. Postherpetic neuralgia: The importance of preventing this intractable end-stage disorder. J Infect Dis 1998;178(Suppl 1):S91-S94.

46. Smith KJ, Roberts MS. Antiviral therapies for herpes zoster infections: Are they economically justifiable? Pharmacoeconomics 2000;2:95-104.

47. Dworkin RH, Johnson RW, Breuer J et al. Recommendations for the management of herpes zoster. Clin Infect Dis 2007;44(Suppl 1):S1-S26. 